\title{
Caracterização nutricional e sociodemográfica de lactantes: uma revisão sistemática
}

\author{
Nutritional and sociodemographic characteristics \\ of nursing mothers: a systematic review
}

\author{
Larissa Bueno Ferreira ${ }^{1}$ \\ Ingrid Tatiana Oliveira de $\mathrm{Nea}^{1}$ \\ Taciana Maia de Sousa ${ }^{1}$ \\ Luana Caroline dos Santos ${ }^{1}$
}

${ }^{1}$ Escola de Enfermagem, Universidade Federal de Minas Gerais. Av. Alfredo Balena 190/324, Santa Efigênia. 30130-100 Belo Horizonte MG Brasil.

labuenoferreira@gmail.com
Abstract A systematic review of nutritional and sociodemographic characteristics of nursing mothers, using the published literature from 2004 to 2014 in the Biblioteca Virtual de Saude and Medline databases. Using the following key words, 561 articles were identified: "Human Milk" and "Milk Banks"; keywords: "Profile," "Nutritional," "Milk Donor," "Nursing Mother" and "Nursing Mothers"; available abstracts; date and language. From these, $84.1 \%$ were excluded for not dealing with the established subject, $1.8 \%$ for being a review and $7.5 \%$ for the unavailability of abstracts for study. Thirty-seven articles were selected, of which $18.9 \%$ analyzed the profile of the HM donor. Of the total, $89.2 \%$ reported age, the majority between 20 and 30 years of age. The level of schooling was mentioned in $32.4 \%$ ranging from elementary school and higher education. With respect to income, a higher prevalence of low socioeconomic status was observed. About the nutritional status, $45.9 \%$ of the studies measured the body mass index and 29.4\% reported the predominance of excess weight. There were deficiencies, particularly of vitamin $A$, with frequencies between $9 \%$ and 26\%. A majority of young adult mothers, varied schooling, low income and nutritional deficiencies were noted, demanding interventions to improve health and increase HM donations.

Key words Health profile, Maternal nutrition, Donations, Human milk
Resumo Revisão sistemática da caracterização nutricional e sociodemográfica de lactantes, por meio da literatura publicada entre 2004-2014, indexadas na Biblioteca Virtual de Saúde e Medline. Foram identificados 561 artigos com os descritores: "Leite Humano" e "Bancos de Leite"; palavras-chave: "Perfil", "Nutricional", "Doadoras de leite", "Nutriz" e "Nutrizes"; resumos disponíveis; data e idioma. Destes, 84,1\% foram excluídos por não atenderem a temática, 1,8\% por se tratarem de revisão e 7,5\% por estarem indisponíveis para consulta. Foram selecionados 37 artigos, dos quais, 18,9\% analisaram o perfil da doadora de LH. Do total, 89,2\% referiram a idade, maioria entre 20 e 30 anos. Nível de escolaridade foi mencionado em 32,4\%, variando entre ensino fundamental e superior. Quanto à renda, encontrou-se predominância de baixo nível socioeconômico. No âmbito nutricional, 45,9\% dos estudos avaliaram o Índice de Massa Corporal e 29,4\% o relataram na faixa de excesso de peso. Observaram-se carências, sobretudo de vitamina $A$, com as frequências de inadequações entre 9-26\%. Observou-se predominância de adultas jovens, escolaridade variada, baixa renda e deficiências nutricionais demandando intervenções para contribuir na melhoria da saúde e aumento das doações de LH. Palavras-chave Perfil de saúde, Nutrição materna, Doações, Leite humano 


\section{Introdução}

O ato de amamentar está intimamente relacionado ao processo de doação de leite humano (LH), visto que, quando a mulher vivencia a experiência da maternidade e da amamentação, ela pode ser doadora desse fluido vital. As doações de leite viabilizam e favorecem a manutenção do aleitamento materno (AM) para lactentes que, por motivos clínicos, não disponham do aleitamento ao seio. Assim, a nutriz doadora exerce um papel fundamental enquanto agente de manutenção dessa prática ${ }^{1}$.

No que diz respeito à lactante que decide doar seu leite, essa é considerada apta quando possui excesso de leite materno (LM). Ainda, deve apresentar exames pré ou pós-natal sem alterações que impeçam a doação, não fumar mais que dez cigarros ao dia, não fazer uso de álcool e drogas ilícitas, não usar medicamentos incompatíveis com a amamentação e possuir bom estado geral de saúde².

Além do adicional de LM disponível para doação, alguns fatores sociodemográficos podem afetar o AM e consequentemente impactar sobre a doação. É conhecido que mães mais jovens amamentam mais seus filhos em comparação às mais velhas. Adicionalmente nota-se também que as lactantes com menor grau de instrução amamentam com mais frequência do que aquelas com nível superior, embora se observe uma tendência crescente da prática do AM entre as mulheres mais instruídas e com maior nível socioeconômi$\mathrm{Co}^{3}$.

Em contrapartida, o estado nutricional inadequado, a saber, a obesidade, parecem desfavorecer o processo de doação. Esse agravo pode influenciar negativamente o estabelecimento da lactação, em virtude de dificuldade de posicionamento do bebê na amamentação e fatores hormonais, já que mulheres com excesso de peso apresentam menor resposta à prolactina nas primeiras 48 horas pós -parto, culminando em atraso na lactogênese e maior probabilidade de desmame precoce. Tal situação é preocupante, visto que o excesso de peso se tornou um problema recorrente após o parto, resultado do exagerado ganho ponderal durante a gestação e pelas elevadas prevalências de excesso de peso no período pré-gestacional ${ }^{4,5}$.

Ainda no âmbito nutricional, outro problema frequente na lactação são as carências nutricionais, pois a demanda de nutrientes neste período, indispensável para a adequada produção de leite e manutenção da saúde materna, é maior. Dentre os agravos mais comuns, estão a deficiência de vitamina A (DVA) e anemia ferropriva, que compreende um dos maiores problemas de saúde pública neste grupo ${ }^{6-9}$.

Diante do exposto, torna-se relevante investigar o perfil de saúde das lactantes de modo a subsidiar a formulação de estratégias para melhoria da saúde materna e ações que possam contribuir para o incremento e qualidade das doações de LH. Assim, o presente trabalho objetivou realizar uma revisão sistemática acerca do perfil nutricional e sociodemográfico de lactantes e seu possível impacto sobre a doação de LM.

\section{Metodologia}

Trata-se de uma revisão sistemática da literatura pautada em bases de dados do sítio da Biblioteca Virtual de Saúde (BVS): Literatura Latino-Americana e do Caribe em Ciências da Saúde (Lilacs), Índice Bibliográfico Espanhol de Ciências da Saúde (IBECS) e Scientific Eletronic Library Online (SciELO). Adicionalmente, foram pesquisadas publicações na base de dados Medline (National Library of Medicine) por meio do PubMed.

Para a pesquisa em ambas as bases de dados (BVS e Medline), foram adotados os descritores: Leite Humano e Bancos de Leite, e as palavras chaves: Perfil, Nutricional, Doadoras de leite, Nutriz e Nutrizes, bem como suas respectivas traduções para a língua inglesa e espanhola. Ressalta-se que não foram utilizados operadores boleanos.

Inicialmente, a seleção dos manuscritos, realizada a partir da leitura do título e resumo, e a exclusão daqueles indexados em mais de uma base de dados foi realizada pela autora principal, respeitando os critérios de inclusão e exclusão pré-determinados. Em seguida, a leitura na íntegra dos artigos remanescentes, e a segunda análise dos estudos foi realizada por dois investigadores independentes. As eventuais discordâncias foram avaliadas e posteriormente chegou-se a um consenso.

Foram selecionados artigos publicados na última década (2004-2014) em idioma inglês, português ou espanhol, cujos resumos estavam disponíveis para consulta. Os investigadores contataram os autores dos resumos indisponíveis, a fim de se obter as informações necessárias. A partir disso, foi realizada uma leitura das sinopses dos manuscritos, selecionando-se aqueles compatíveis com a temática proposta. Foi realizada a exclusão de resumos em duplicata em ambas as bases de pesquisa e aqueles cujos autores não retornaram o contato. 
Por fim, foi realizada a leitura completa dos manuscritos elegidos. Os artigos de revisão e aqueles que, apesar dos rigorosos critérios de seleção (período da publicação, descritores, viabilidade), não abordavam a temática proposta ou se encontravam indisponíveis, foram excluídos.

No tocante à qualidade metodológica, todos os trabalhos fizeram uso de ferramentas validadas para avaliar as variáveis selecionadas e análises estatísticas adequadas.

\section{Resultados e discussão}

Foram encontrados 561 artigos, a partir da revisão inicial, dos quais $398(70,9 \%)$ correspondiam à base de dados Medline e $163(29,1 \%)$ à BVS. Identificou-se que $472(84,1 \%)$ não atendiam a temá- tica em questão, abordando itens como a nutrição de animais e períodos que antecedem a lactação, sendo excluídos. Além disso, 42 (7,5\%) não foram avaliados em virtude da indisponibilidade dos resumos para consulta. Foram excluídos também os artigos de revisão $(\mathrm{n}=10)$, que corresponderam a $1,8 \%$ de todos os manuscritos pesquisados. Para a análise final, foram incluídos 37 artigos que contemplavam lactantes, e, desses, 7 (18,9\%) retrataram o perfil nutricional e/ou sociodemográfico da nutriz doadora de LH (Figura 1).

Identificaram-se nos artigos selecionados as variáveis que se relacionam ao tema proposto por esta revisão: dados sociodemográficos e nutricionais, a saber, antropometria, consumo alimentar e avaliação sérica de micronutrientes (Quadro 1). Posteriormente, as principais características acerca do perfil das lactantes foram descritas. Tabela 1.

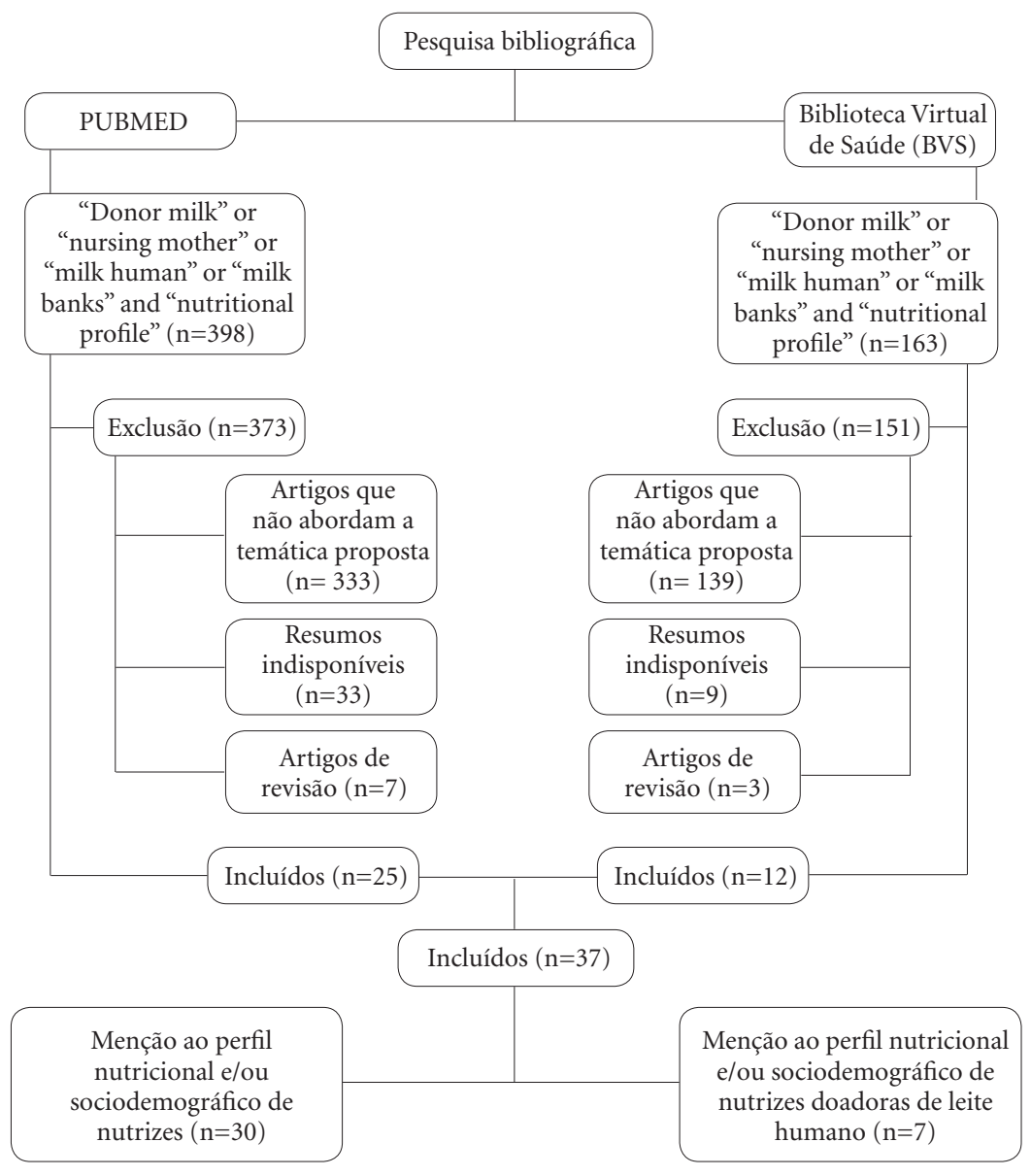

Figura 1. Fluxograma do processo de seleção dos artigos. 
Quadro 1. Síntese dos artigos conforme perfil estudado.

\begin{tabular}{|c|c|c|c|c|c|}
\hline \multirow[b]{2}{*}{ Autores } & \multirow[b]{2}{*}{ Público alvo } & \multicolumn{4}{|c|}{ Características } \\
\hline & & Sociodemográficas & Antropométricas & $\begin{array}{l}\text { Consumo } \\
\text { alimentar }\end{array}$ & \begin{tabular}{|c|} 
Avaliação \\
sérica de \\
micronutrientes \\
\end{tabular} \\
\hline Antonakou et al. & $\begin{array}{l}\text { Nutriz não doadora } \\
\qquad(\mathrm{n}=64)\end{array}$ & $\mathrm{X}$ & & $\mathrm{X}$ & \\
\hline Azeredo et al. & $\begin{array}{l}\text { Nutriz não doadora } \\
\qquad(\mathrm{n}=50)\end{array}$ & $\mathrm{X}$ & $\mathrm{X}$ & & \\
\hline Bachour et al. ${ }^{10}$ & $\begin{array}{l}\text { Nutriz não doadora } \\
(\mathrm{n}=66)\end{array}$ & $\mathrm{X}$ & $\mathrm{X}$ & & \\
\hline Cherop et al. ${ }^{11}$ & $\begin{array}{l}\text { Nutriz não doadora } \\
\qquad(\mathrm{n}=384)\end{array}$ & $\mathrm{X}$ & & & \\
\hline Colomina et al. & $\begin{array}{l}\text { Nutriz doadora } \\
\qquad(\mathrm{n}=168)\end{array}$ & $\mathrm{X}$ & & & \\
\hline $\begin{array}{l}\text { Correia-Santos } \\
\text { et al. }\end{array}$ & $\begin{array}{l}\text { Nutriz não doadora } \\
\qquad(\mathrm{n}=36)\end{array}$ & $\mathrm{X}$ & $\mathrm{X}$ & & $\mathrm{X}$ \\
\hline Dias et al. & $\begin{array}{l}\text { Nutriz doadora } \\
\quad(\mathrm{n}=48)\end{array}$ & $\mathrm{X}$ & & & \\
\hline $\begin{array}{l}\text { Dimenstein } \\
\text { et al. }\end{array}$ & $\begin{array}{l}\text { Nutriz não doadora } \\
\qquad(\mathrm{n}=30)\end{array}$ & $\mathrm{X}$ & $\mathrm{X}$ & & $\mathrm{X}$ \\
\hline $\begin{array}{c}\text { Dimenstein et } \\
\text { al. }^{12}\end{array}$ & $\begin{array}{l}\text { Nutriz não doadora } \\
\qquad(\mathrm{n}=72)\end{array}$ & $\mathrm{X}$ & & & $\mathrm{X}$ \\
\hline Ettyang et al. ${ }^{13}$ & $\begin{array}{l}\text { Nutriz não doadora } \\
(\mathrm{n}=10)\end{array}$ & $\mathrm{X}$ & $\mathrm{X}$ & & $\mathrm{X}$ \\
\hline Galvão et al. & $\begin{array}{l}\text { Nutriz doadora } \\
\qquad(\mathrm{n}=11)\end{array}$ & $\mathrm{X}$ & & & \\
\hline Harit et al. ${ }^{14}$ & $\begin{array}{l}\text { Nutriz não doadora } \\
\qquad(\mathrm{n}=299)\end{array}$ & & $\mathrm{X}$ & & \\
\hline Lira et al. & $\begin{array}{l}\text { Nutriz não doadora } \\
\qquad(\mathrm{n}=97)\end{array}$ & $\mathrm{X}$ & & & $\mathrm{X}$ \\
\hline Lopes et al. & $\begin{array}{l}\text { Nutriz não doadora } \\
(\mathrm{n}=46)\end{array}$ & $\mathrm{X}$ & $\mathrm{X}$ & $\mathrm{X}$ & \\
\hline Lubetzky et al. ${ }^{15}$ & $\begin{array}{l}\text { Nutriz não doadora } \\
\qquad(\mathrm{n}=55)\end{array}$ & $\mathrm{X}$ & $\mathrm{X}$ & & \\
\hline Marín et al. & $\begin{array}{l}\text { Nutriz não doadora } \\
(\mathrm{n}=46)\end{array}$ & $\mathrm{X}$ & $\mathrm{X}$ & $\mathrm{X}$ & \\
\hline Maru et al. ${ }^{17}$ & $\begin{array}{l}\text { Nutriz não doadora } \\
(\mathrm{n}=45)\end{array}$ & $\mathrm{X}$ & $\mathrm{X}$ & & \\
\hline Mello-Neto et al. & $\begin{array}{l}\text { Nutriz doadora } \\
\qquad(\mathrm{n}=136)\end{array}$ & $\mathrm{X}$ & $\mathrm{X}$ & & $\mathrm{X}$ \\
\hline Nakamori et al. & $\begin{array}{l}\text { Nutriz não doadora } \\
\qquad(\mathrm{n}=60)\end{array}$ & $\mathrm{X}$ & $\mathrm{X}$ & $\mathrm{X}$ & $\mathrm{X}$ \\
\hline Neves et al. & $\begin{array}{c}\text { Nutriz doadora }(\mathrm{n}=4) \\
\text { Nutriz não doadora } \\
(\mathrm{n}=3)\end{array}$ & $\mathrm{X}$ & & & \\
\hline $\begin{array}{c}\text { Olafsdottir et } \\
\text { al. }^{18}\end{array}$ & $\begin{array}{l}\text { Nutriz não doadora } \\
(\mathrm{n}=77)\end{array}$ & $\mathrm{X}$ & $\mathrm{X}$ & $\mathrm{X}$ & \\
\hline Örün et al. & $\begin{array}{l}\text { Nutriz não doadora } \\
\qquad(\mathrm{n}=144)\end{array}$ & $\mathrm{X}$ & & $\mathrm{X}$ & \\
\hline Qiao et al. ${ }^{19}$ & $\begin{array}{l}\text { Nutriz não doadora } \\
\qquad(\mathrm{n}=90)\end{array}$ & & & $\mathrm{X}$ & \\
\hline Quinn et al. & $\begin{array}{l}\text { Nutriz não doadora } \\
\qquad(\mathrm{n}=102)\end{array}$ & $\mathrm{X}$ & $\mathrm{X}$ & $\mathrm{X}$ & \\
\hline Ribeiro et al. & $\begin{array}{l}\text { Nutriz não doadora } \\
\qquad(\mathrm{n}=86)\end{array}$ & $\mathrm{X}$ & & & $\mathrm{X}$ \\
\hline
\end{tabular}


Quadro 1. continuação

\begin{tabular}{|c|c|c|c|c|c|}
\hline \multirow[b]{2}{*}{ Autores } & \multirow[b]{2}{*}{ Público alvo } & \multicolumn{4}{|c|}{ Características } \\
\hline & & Sociodemográficas & Antropométricas & $\begin{array}{l}\text { Consumo } \\
\text { alimentar }\end{array}$ & $\begin{array}{c}\text { Avaliação } \\
\text { sérica de } \\
\text { micronutrientes }\end{array}$ \\
\hline Sánchez et al. & $\begin{array}{l}\text { Nutriz não doadora } \\
\qquad(\mathrm{n}=39)\end{array}$ & $\mathrm{X}$ & $\mathrm{X}$ & $\mathrm{X}$ & \\
\hline Santos et al. & $\begin{array}{l}\text { Nutriz doadora } \\
\qquad(\mathrm{n}=91)\end{array}$ & $\mathrm{X}$ & & & \\
\hline Severi et al. & $\begin{array}{l}\text { Nutriz não doadora } \\
(\mathrm{n}=312)\end{array}$ & $\mathrm{X}$ & & & $\mathrm{X}$ \\
\hline Shashiraj et al. ${ }^{20}$ & $\begin{array}{l}\text { Nutriz não doadora } \\
(\mathrm{n}=200)\end{array}$ & $\mathrm{X}$ & $\mathrm{X}$ & & \\
\hline Soares et al. & $\begin{array}{l}\text { Nutriz não doadora } \\
\quad(\mathrm{n}=40)\end{array}$ & $\mathrm{X}$ & & & \\
\hline Souza et al. & $\begin{array}{l}\text { Nutriz não doadora } \\
(\mathrm{n}=196)\end{array}$ & $\mathrm{X}$ & & & \\
\hline $\begin{array}{c}\text { Szlagatys- } \\
\text { Sidorkiewicz et } \\
\text { al. }^{21} \\
\end{array}$ & $\begin{array}{l}\text { Nutriz não doadora } \\
\qquad(\mathrm{n}=156)\end{array}$ & $\mathrm{X}$ & $\mathrm{X}$ & $\mathrm{X}$ & \\
\hline $\begin{array}{c}\text { Szlagatys- } \\
\text { Sidorkiewicz et } \\
\text { al. }^{21} \\
\end{array}$ & $\begin{array}{l}\text { Nutriz não doadora } \\
\qquad(\mathrm{n}=24)\end{array}$ & $\mathrm{X}$ & & & \\
\hline Tavares et al. & $\begin{array}{l}\text { Nutriz não doadora } \\
\qquad(\mathrm{n}=75)\end{array}$ & $\mathrm{X}$ & $\mathrm{X}$ & $\mathrm{X}$ & \\
\hline $\begin{array}{c}\text { Tokuşoğlu et } \\
\text { al. }^{22}\end{array}$ & $\begin{array}{c}\text { Nutriz não doadora } \\
\qquad(\mathrm{n}=92)\end{array}$ & $\mathrm{X}$ & $\mathrm{X}$ & $\mathrm{X}$ & \\
\hline Valentine et al. ${ }^{23}$ & $\begin{array}{l}\text { Nutriz doadora } \\
\qquad(\mathrm{n}=21)\end{array}$ & $\mathrm{X}$ & & $\mathrm{X}$ & \\
\hline $\begin{array}{l}\text { Zhixiong Shi et } \\
\text { al. }^{24}\end{array}$ & $\begin{array}{l}\text { Nutriz não doadora } \\
\qquad(\mathrm{n}=103)\end{array}$ & $\mathrm{X}$ & $\mathrm{X}$ & $\mathrm{X}$ & \\
\hline
\end{tabular}

Observou-se que as variáveis de maior abordagem nos artigos em estudo foram as sociodemográficas, sendo estas mencionadas em 91,9\% $(\mathrm{n}=34)$. As informações nutricionais foram analisadas em 78,4\% $(n=29)$, enquanto 5,4\% ( $n=$ 2) dos artigos analisados contemplaram todas as características pesquisadas por esta revisão (Tabela 1$)$.

\section{Características sociodemográficas}

Do ponto de vista sociodemográfico foram detectadas informações referentes à idade, escolaridade, renda e paridade.

\section{Idade}

Dos artigos analisados, 89,2\% ( $\mathrm{n}=33)$ mencionaram a idade das lactantes e observou-se predominância de adultas jovens (20 a 30 anos $)^{25}$.
Em um hospital universitário de Maringá (PR), a maioria das nutrizes avaliadas $(41,7 \%)$ tinha entre 20 e 29 anos $^{26}$. Tal resultado é semelhante aos achados de Santos et al. ${ }^{27}$, que encontraram maioria das mulheres $(28,6 \%)$ entre 24 e 28 anos e, também ao estudo de Mello-Neto et al..$^{28}$, no qual mais da metade das doadoras de LH (54,4\%) tinha entre 20 e 26 anos de idade.

Alguns trabalhos investigaram a idade média, como os realizados por Nakamori et al. ${ }^{8}$, e Örün et al. ${ }^{29}$, e esta foi de 25 anos em ambos. Em um estudo realizado com lactantes doadoras de um Banco de Leite Humano (BLH) na Espanha, encontrou-se média de 33,1 anos $^{30}$. Sánchez et al. ${ }^{31}$, já detectaram maiores médias de idade, 33,4 anos.

Apesar desta predominância já esperada de mulheres jovens, por se tratar do período do ciclo de vida mais propício para a gestação, cabe destacar que a idade parece não influenciar dire- 
Tabela 1. Principais características nutricionais referentes às lactantes.

\begin{tabular}{|c|c|c|}
\hline Variável Estudada & $\begin{array}{l}\text { Percentual (\%) de } \\
\text { número de Estudos }\end{array}$ & Síntese dos resultados \\
\hline Antropometria & $45,9(\mathrm{n}=17)$ & $\begin{array}{l}35,3 \% \text { encontraram média na faixa de eutrofia } \\
(\mathrm{n}=6) \text { e } 17,6 \% \text { na faixa de sobrepeso }(\mathrm{n}=3) \\
23,5 \% \text { encontraram maior prevalência de } \\
\text { classificação para excesso de peso }(n=4) \text { e } 23,5 \% \\
\text { para eutrofia }(n=4) .\end{array}$ \\
\hline Ingestão Média de Energia & $10,8(n=4)$ & $\begin{array}{l}50 \% \text { encontraram média }>2000 \mathrm{kcal}(\mathrm{n}=2), 25 \% \\
\text { média }<1500 \mathrm{kcal}(\mathrm{n}=1) \text { e } 25 \% \text { média entre } 1500 \text { e } \\
2000 \mathrm{kcal}(\mathrm{n}=1)\end{array}$ \\
\hline Ingestão Média de Proteína & $13,5(n=5)$ & $\begin{array}{l}100 \% \text { encontraram percentual médio dentro da } \\
\text { recomendação }(10 \text { a } 35 \%)(n=5)\end{array}$ \\
\hline $\begin{array}{l}\text { Ingestão Média de } \\
\text { Carboidratos }\end{array}$ & $13,5(n=5)$ & $\begin{array}{l}80 \% \text { encontraram percentual médio dentro da faixa } \\
\text { recomendada }(45 \text { a } 65 \%)(n=4) \text { e } 20 \% \text { abaixo }(n=1)\end{array}$ \\
\hline Ingestão Média de Gorduras & $16,2(n=6)$ & $\begin{array}{l}50 \% \text { encontraram percentual médio dentro da } \\
\text { faixa recomendada }(10 \text { a } 35 \%)(n=3), 16,7 \% \text { abaixo } \\
(n=1) \text { e } 33,3 \% \text { acima }(n=2) \text {. }\end{array}$ \\
\hline Carência Nutricional & $13,5(n=5)$ & $\begin{array}{l}40 \% \text { encontraram DVA }(\mathrm{n}=2) ; 40 \% \text { anemia }(\mathrm{n}=2) \\
20 \% \text { hemoglobina abaixo da faixa adequada }(12 \mathrm{a} \\
16,5 \mathrm{~g} / 100 \mathrm{ml})(\mathrm{n}=1)\end{array}$ \\
\hline
\end{tabular}

DVA: Deficiência de Vitamina A.

tamente a doação de $\mathrm{LM}^{25,27}$. No entanto, essa é uma variável importante a se considerar em ações relacionadas à doação, principalmente pelas outras variáveis a ela associadas, de maneira direta ou indireta, tal como a escolaridade e renda.

\section{Escolaridade}

O nível de escolaridade foi mencionado em $32,4 \%(n=12)$ dos artigos, sendo detectada ampla variação em relação ao grau de instrução das lactantes com maior prevalência de baixa escolaridade. O estudo realizado por Örün et al. $^{29}$, mostrou que a maioria das nutrizes tinha menos que 9 anos de estudo, similar ao encontrado por Galvão et al. ${ }^{1}$. Adicionalmente, em outro estudo, com 312 lactantes do Uruguai encontrou-se média de escolaridade inferior a 9 anos, a saber, 7,7 anos para as nutrizes adolescentes e 8,1 anos para as adultas ${ }^{32}$.

Em contraponto, alguns achados apontaram mais tempo de escolaridade, como o de Tavares et al..$^{33}$, e Quinn et al. ${ }^{34}$, que encontraram médias de 10 e 9,8 anos de estudos, respectivamente. Além disso, Colomina et al. ${ }^{30}$ observaram que a maioria das lactantes $(65,3 \%)$ apresentava educação universitária. Essas diferenças quanto ao nível de educação podem ocorrer em função da idade, visto que os achados que apontam maior grau de instrução referem-se também a maiores médias etárias ${ }^{30,34,35}$.

Apesar da disparidade no que se refere à escolaridade das nutrizes, os estudos apontam tendência crescente de amamentação entre mulheres com maior nível de educação, provavelmente pelo maior suporte familiar e social, acesso facilitado aos serviços de atenção à saúde e à inserção formal no mercado de trabalho. Ademais, o grau de instrução da nutriz pode interferir na compreensão da mensagem sobre a prática do AM e, portanto, na decisão de doação do LM $^{27,36}$. Assim, estratégias de incentivo à doação devem conseguir abarcar os diferentes níveis de escolaridade, com ações de complexidade distintas.

\section{Renda}

A renda familiar foi mencionada por 10,8\% $(\mathrm{n}=4)$ dos autores que apontaram perfil predominante de nutrizes com baixo nível socioeconômico (renda familiar mensal per capita de meio salário mínimo e, no máximo, até três salários mínimos $)^{37}$. Em estudo realizado no Rio de Janeiro, Souza et al. ${ }^{9}$, notaram que a renda familiar per capita era inferior a um salário em 52,5\% da amostra. Já em estudo conduzido por Galvão et 
al. ${ }^{1}$, na cidade de Fortaleza, a maioria das nutrizes $(45,5 \%)$ apresentava renda familiar entre 2 e 4 salários mínimos.

Em São Paulo, verificou-se que a maior parte $(59,3 \%)$ das lactantes avaliadas tinha renda familiar de até 2 salários mínimos. Tal situação parece ocorrer em função do local do estudo, visto que a maioria $(87 \%)$ das mulheres avaliadas residia na Zona Leste da cidade, que compreende uma das regiões mais pobres, de pior renda média familiar e menor nível de atividade econômica ${ }^{38}$.

Diante disso, cabe ressaltar que o menor nível socioeconômico pode determinar o acesso desigual aos serviços de saúde. Mulheres de baixa renda são mais desprovidas de assistência durante o pré-natal e puerpério e, consequentemente, de informações básicas para o desfecho satisfatório do ciclo reprodutivo. É nos serviços de saúde também que lactantes recebem informações e devem ser sensibilizadas quanto ao processo de doação de LH denotando, portanto, a importância do acesso a esses locais ${ }^{4,39}$. Adicionalmente, informações sobre a doação também precisam ser veiculadas com frequência nos meios de comunicação mais acessíveis, a fim de alcançar todas as nutrizes, independente do nível socioeconômico avaliado.

\section{Paridade}

Em relação à paridade, 18,9\% $(\mathrm{n}=7)$ dos artigos a averiguaram e houve predominância de primíparas $(57,1 \%)$. Em estudo com 48 lactantes doadoras, 62,5\% eram primíparas, enquanto em um Hospital Universitário de Londrina (Paraná) este percentual era de $56 \%{ }^{27}$. Já os achados de Ribeiro et al. ${ }^{40}$, e Lira et al. ${ }^{41}$, apontaram que $62,5 \%$ e $52 \%$ das lactantes eram multíparas, respectivamente.

As diferenças encontradas em relação à paridade podem ocorrer em função da idade das lactantes, uma vez que, geralmente, mulheres com maior número de filhos são também mais velhas. Os estudos de Dias et al. ${ }^{26}$, e Santos et al. ${ }^{27}$, que encontraram alta prevalência de primíparas, comprovam essas ideias, pois a maioria das nutrizes (64,6 e 60,5\%, respectivamente) eram mulheres jovens. Ainda referente à paridade, em estudo revisado por Castro et al. ${ }^{4}$ observou-se que as multíparas são mais frequentes nas classes sociais mais baixas e com menor nível de escolaridade.

No que tange ao número de filhos, há que se pensar que as mulheres multíparas já vivenciaram a experiência do AM, sentindo-se mais con- fiantes no processo da amamentação. Por outro lado, cabe salientar que a primiparidade é fator de risco para as intercorrências mamárias, em virtude da inexperiência na prática da lactação. Diante disso, mulheres primíparas buscam auxílio nos BLH com maior frequência, consequentemente têm mais acesso a informação acerca dos benefícios da doação e tornam-se, portanto, mais sujeitas a doarem o seu leite $e^{26,27}$.

Em síntese, o perfil sociodemográfico identificado denota a importância de estratégias específicas, como a ampliação da assistência médica e de ações políticas que aumentem a cobertura dessas mulheres, além das existentes, que favoreçam a homogeneização do acesso aos serviços de saúde, independente das condições socioeconômicas. Nesse contexto, o maior acesso a esses locais determinará esclarecimentos importantes que podem viabilizar o incremento das doações de LH.

\section{Características Nutricionais}

\section{Antropometria}

Dos 37 estudos selecionados, 45,9\% $(\mathrm{n}=17)$ avaliaram o estado nutricional por meio do Índice de Massa Corporal (IMC). Destes, 29,4\% (n = 5) relataram IMC médio na faixa de classificação para excesso de peso. Entre esses achados estão Tavares et al. ${ }^{33}$, que investigaram mulheres a partir do $28^{\circ}$ dia pós parto e encontraram IMC médio de $26,4 \mathrm{~kg} / \mathrm{m}^{2}$. Além deste, Lopes et al. ${ }^{42} \mathrm{e}$ Sanchez et al. ${ }^{31}$, que incluíram lactantes a partir de um mês após o parto, encontraram IMC médio de 27,4 e $26,2 \mathrm{~kg} / \mathrm{m}^{2}$, respectivamente.

Cabe ressaltar que a interpretação desses dados deve ser realizada com cautela. O tempo de seguimento desses estudos são considerados curtos (até 6 semanas pós parto), sendo este o período em que ocorre o reequilíbrio hidroeletrolítico gradual, em que a água extracelular e extravascular aumentada na gestação diminui, e o volume circulante retorna a valores pré-gravídicos. Somente depois desse período, é que a retenção de peso pós-parto pode ser atribuída ao aumento de gordura corporal materna adquirida durante a gestação, bem como ao aumento do tecido mamário ${ }^{43}$.

Apesar das limitações para a avaliação desses dados, destaca-se que o pós-parto é um período que vem sendo associado ao excesso de peso em virtude do elevado ganho ponderal durante a gestação. No que concerne a isso, estudos longitudinais revisados por Castro et al. ${ }^{4}$ apontaram valores elevados de retenção ponderal pós-parto, 
sendo as frequências entre $14 \%$ e $25,6 \%$, nas populações estudadas.

Assim, o acompanhamento adequado da gestante durante o pré-natal é essencial para o controle ponderal durante a gestação, visto que este é um preditor para obesidade futura, além de favorecer o processo de doação de LH pela interferência na lactogênese $e^{5,6}$.

\section{Ingestão energética e de macronutrientes}

Dos 15 artigos que avaliaram o consumo alimentar, 33,3\% (n=5) analisou a adequação da ingestão de carboidrato $(\mathrm{CHO}), 33,3 \%(\mathrm{n}=5)$, de proteína (PTN), 40\% $(\mathrm{n}=6)$, de lipídio (LIP) e 26,7\% ( $n=4)$, o consumo calórico (Tabela 1$)$.

Quanto aos estudos que avaliaram a ingestão de macronutrientes, em sua maioria encontraram valores médios dentro da faixa de recomendação: 10 a 35\%, para PTN; 45 a 65\%, para CHO; e 20 a $35 \%$, para LIP ${ }^{44}$.

Adicionalmente, de acordo com o Institute of Medicine ${ }^{44}$ o consumo alimentar da nutriz deve oferecer, no mínimo, $1800 \mathrm{kcal}$ ao dia. Dos artigos que mencionaram a ingestão energética, 75\% $(\mathrm{n}=3)$ encontraram resultados superiores a esta recomendação. No entanto, dietas com menos de 1500 kcal são desaconselhadas ${ }^{44}$, por não atenderem as necessidades nutricionais da mulher nesse período $^{34}$.

Entre 75 nutrizes paulistas avaliadas, por meio de Recordatório Alimentar de 24 horas e Questionário de Frequência Alimentar, o valor energético médio ingerido por dia foi de $2.233 \mathrm{kcal}^{33}$. Sánchez et al. ${ }^{31}$ investigaram o consumo calórico utilizando os mesmos inquéritos nutricionais e encontraram média de 2.340,5 kcal/dia. Esses valores se aproximam das necessidades energéticas de uma nutriz de referência, com peso e altura apropriados, que é de aproximadamente $2.300 \mathrm{kcal}^{44}$.

Resultados divergentes, no entanto, foram observados por Quinn et al. ${ }^{34}$ e Lopes et al. ${ }^{42}$, que referiram médias de 1411,3 kcal e 3392,1 kcal, respectivamente. Tais discrepâncias demandam atenção, pois, na lactação, há necessidade de maior aporte energético visando garantir a produção de LM, porém o excesso não é desejável. Recomenda-se em média um adicional de 500 $\mathrm{kcal} /$ dia nos primeiros seis meses e de $400 \mathrm{kcal} /$ dia nos meses subsequentes. $\mathrm{O}$ consumo calórico insuficiente pode acarretar danos à saúde materna, como, por exemplo, a deficiência de nutrientes, enquanto o excessivo pode favorecer o acúmulo de gordura e retenção ponderal pós-parto ${ }^{4}$.

A dieta materna sofre influência de fatores demográficos, socioeconômicos, culturais e so- ciais. A idade, por exemplo, interfere no consumo energético, visto que mulheres mais velhas apresentam menor consumo de calorias em relação às mais jovens ${ }^{33}$. Isso aponta a demanda por atenção individualizada, no que se refere a avaliação nutricional e orientações especificas para uma alimentação equilibrada a fim de estimular a doação de LH.

\section{Ingestão dietética e níveis séricos de micronutrientes}

Em relação à avaliação de micronutrientes, poucos estudos referiram essas informações $10,8 \%(\mathrm{n}=4)$ com dados dietéticos e 32,4\% ( $\mathrm{n}=$ 12) com parâmetros séricos. Quanto a ingestão, os estudos selecionados por este trabalho investigaram ferro, zinco e cálcio (Tabela 2), possivelmente pelas implicações destes nutrientes para a saúde da nutriz.

A média de ingestão de ferro, zinco e cálcio foi aquém da recomendada em todas as investigações $^{8,34,42}$. Porém, não há evidências de que os níveis desses micronutrientes no LH dependam da dieta materna, em vista da manutenção da homeostase realizada pelo organismo. Assim, para esses nutrientes a qualidade da dieta da mãe não afeta a qualidade de seu leite, mas é importante que a nutriz tenha níveis adequados para manter a sua própria saúde, favorecendo o processo de doação do $\mathrm{LH}^{45}$.

Tavares et al. ${ }^{33}$ avaliaram a ingestão de cálcio e esta foi abaixo da recomendação em $64 \%$ das participantes. Lopes et al. ${ }^{42}$ também observaram valores inferiores às recomendações para este nutriente, com média de 659,8 $\pm 307,1 \mathrm{mg}$. Atenção especial deve ser dada a este nutriente, porque adaptações fisiológicas ocorrem no corpo durante a lactação, a fim de assegurar uma transferência adequada de cálcio para a glândula mamária. Tais adaptações podem levar à perda temporária de massa óssea, à elevação da absorção de cálcio e redução da excreção urinária ${ }^{46}$.

A perda de massa óssea durante a lactação pode ser transitória e não aumenta o risco de osteoporose ou fraturas. Porém, essas situações podem ocorrer se a ingestão materna de cálcio for inferior a $500 \mathrm{mg} / \mathrm{dia}$. Assim, a nutriz deve-se ater para o consumo de alimentos fonte desse micronutriente, como leite e derivados ${ }^{45}$.

O cálcio sérico foi analisado por 1 (2,7\%) estudo, conduzido por Correia Santos et al. ${ }^{46}$, e a concentração média encontrada estava dentro do intervalo normal (entre 2,2 e 2,5 mmol/L), denotando a forte regulação homeostátisca deste mineral. 
Tabela 2. Ingestão Diária Recomendada (RDA) e níveis séricos de micronutrientes recomendados

\begin{tabular}{cccc}
\hline \multirow{2}{*}{ Micronutrientes } & \multicolumn{2}{c}{ RDA (lactantes) } & \multirow{2}{*}{ Níveis séricos } \\
\cline { 2 - 3 } & $\mathbf{1 4 - 1 8 ~ a n o s}$ & $\mathbf{1 9 - 5 0 ~ a n o s}$ & \\
\hline Vitamina A & $1200 \mu \mathrm{\mu g} / \mathrm{d}^{11}$ & $1300 \mu \mathrm{gg} / \mathrm{d}^{11}$ & \multicolumn{2}{c}{$\mu \mathrm{mol} / \mathrm{L}^{37}$} \\
Ferro & $10 \mathrm{mg} / \mathrm{d}^{11}$ & $9 \mathrm{mg} / \mathrm{d}^{11}$ & $>7,2 \mathrm{mmol} / \mathrm{L}^{43}$ \\
Cobre & $1300 \mu \mathrm{\mu g} / \mathrm{d}^{11}$ & $1300 \mu \mathrm{g} / \mathrm{d}^{11}$ & $>10 \mu \mathrm{mol} / \mathrm{L}^{43}$ \\
Zinco & $13 \mathrm{mg} / \mathrm{d}^{11}$ & $12 \mathrm{mg} / \mathrm{d}^{11}$ & $>9,2 \mu \mathrm{mol} / \mathrm{L}^{43}$ \\
Cálcio & $1300 \mathrm{mg} / \mathrm{d}^{21}$ & $1000 \mathrm{mg} / \mathrm{d}^{21}$ & $2,5-2,5 \mathrm{mmol} / \mathrm{L}^{43}$ \\
Vitamina E & $19 \mathrm{mg} / \mathrm{d}^{14}$ & $19 \mathrm{mg} / \mathrm{d}^{14}$ & $>16,2 \mu \mathrm{mol} / \mathrm{L}^{48}$ \\
\hline
\end{tabular}

O nível sérico de outros nutrientes também foi mensurado. Do total, 5 artigos $(41,6 \%)$ avaliaram o retinol, indicador que tem sido amplamente utilizado para identificar as populações em risco de DVA. De acordo com a Organização Mundial da Saúde (OMS), concentrações abaixo de $0,7 \mu \mathrm{mol} / \mathrm{L}$ indicam risco de hipovitaminose A, e a carência desse micronutriente altera sua concentração no $\mathrm{LM}^{28,40,47}$.

Um dos principais fatores que leva a DVA é uma ingestão insuficiente de alimentos de origem animal, como leite e derivados, ovos e fígado, bem como os de origem vegetal ricos em provitamina $\mathrm{A}$, tais como manga, mamão, cenoura, abóbora e moranga ${ }^{40,48}$.

Lira et al. ${ }^{41}$ realizaram uma análise individualizada dos níveis séricos de retinol em 97 puérperas, em Natal (RN), e detectaram prevalência de deficiência subclínica em 15\% da amostra. Já Ribeiro et al. ${ }^{40}$ e Soares et al. ${ }^{47}$ encontraram frequências de inadequação de $9,3 \%$ e $7,5 \%$, respectivamente. Mello-Neto et al..$^{28}$, por sua vez, realizaram o estudo em São Paulo e observaram maior prevalência de DVA: $25,7 \%$ das nutrizes avaliadas, o que corrobora os achados que identificaram maior prevalência dessa deficiência na região Sudeste do país ${ }^{7,48}$. Esses resultados denotam que a DVA não é um problema restrito a regiões de extrema pobreza do Brasil, e que o consumo inadequado está mais relacionado a fatores culturais e hábitos alimentares do que a questões econômicas. Por este motivo, o Programa Nacional de Suplementação de Vitamina A, que antes se concentrava na Região Nordeste, municípios do Vale do Jequitinhonha e Mucuri (Estado de Minas Gerais) e alguns municípios da Amazônia Legal, foi ampliado para todo o País (todos os municípios da Região Norte e Distritos Sanitários Especiais Indígenas e municípios prioritários do Plano Brasil Sem Miséria das regiões Centro-Oeste, Sudeste e Sul) ${ }^{49}$.
Adicionalmente, destaca-se que a idade materna e a paridade apresentam uma relação positiva com os níveis de vitamina A séricos. A lactação prévia proporciona maior mobilização das reservas de retinol, estimulada também pela maior adiposidade materna em multíparas, com posterior transferência à glândula mamária ${ }^{40,41}$.

A concentração de ferro no sangue foi analisada por $3(8,1 \%)$ estudos. Mello Netto et al. ${ }^{28}$ e Correia Santos et al. ${ }^{46}$ encontraram valores médios de acordo com a normalidade $(>7,2$ $\mathrm{mmol} / \mathrm{L}$ ). Na pesquisa conduzida por Nakamori et al. ${ }^{8}$, com lactantes entre 6 e 12 meses pós-parto, a anemia foi encontrada em $39 \%$ das participantes, consequência da ingestão de ferro aquém do recomendado, conforme informações acerca do consumo alimentar investigadas por este estudo.

Esses dados corroboram a deficiência de ferro e a anemia como sendo um grande problema de nível mundial. Existem programas governamentais para amenizá-los, como o Programa Nacional de Suplementação de Ferro que fornece administração profilática de suplementos desse mineral para, além de crianças de 6 a 24 meses de idade, gestantes e mulheres até $3^{\circ}$ mês pós-parto ${ }^{49}$. Todavia, são necessárias mais estratégias para o diagnóstico e tratamento da anemia durante o período da lactação. Sabe-se que as orientações dietéticas constituem intervenções essenciais para estímulo ao consumo de alimentos que contenham ferro de alta biodisponibilidade. Portanto, o acompanhamento nutricional nesse período torna-se fundamental.

A concentração sanguínea de zinco foi investigada em 10,8\% $(n=4)$ dos estudos. CorreiaSantos et al..$^{46}$ e Mello Netto et al. ${ }^{28}$ encontraram os níveis médios adequados $(>9,2 \mu \mathrm{mol} / \mathrm{L})$. Severi et al. ${ }^{32}$ relataram que a prevalência de hipozincemia foi relativamente baixa, sendo 7,3\% em adolescentes e 12,3\% em adultas. Isso pode ser justificado pela maior ingestão de zinco em 
função do alto consumo de produtos cárneos no Uruguai, local onde o estudo foi realizado. Entretanto, Nakamori et al. ${ }^{8}$ encontraram percentual de inadequação em $55,4 \%$ das participantes do estudo, ocorrido em função da baixa ingestão de alimentos fontes de zinco.

Sabe-se que as deficiências de vitamina $\mathrm{A}$, ferro e zinco frequentemente coexistem. Isso acontece, pois há uma interação entre o metabolismo desses micronutrientes. O zinco é um elemento importante para síntese de Retinol Binding Protein (RBP), uma proteína responsável pelo transporte de vitamina A. Assim, níveis sanguíneos reduzidos desse mineral acarretam baixa concentração do retinol sérico. A deficiência de ferro, por sua vez, compromete o funcionamento da mucosa intestinal, resultando em menor absorção da vitamina $\mathrm{A}^{50}$.

Correia-Santos et al. ${ }^{46}$ e Nakamori et al. ${ }^{8}$ também avaliaram a concentração de cobre no plasma. Em relação ao primeiro, foi encontrada média acima dos níveis séricos normais (> $10 \mu \mathrm{mol} / \mathrm{L}$ ). Já Nakamori et al. ${ }^{8}$ constataram que $21,4 \%$ das lactantes apresentavam níveis séricos insuficientes.

A ingestão de cobre parece não comprometer sua concentração no LH, mas o estado nutricional de zinco pode afetar a presença de cobre no leite. Isso ocorre porque a ingestão marginal de zinco durante a lactação aumenta os transportadores de cobre da glândula mamária e altera sua localização, resultando, portanto, em aumento de cobre no $\mathrm{LH}^{8}$.

Em relação aos $2(5,4 \%)$ estudos que avaliaram alfa-tocoferol, conduzidos por Dimenstein et al. ${ }^{51}$, os valores médios encontrados foram adequados, de acordo com a referência adotada pelo estudo ( $>16,2 \mu \mathrm{mol} / \mathrm{L}$ ). Esses resultados indicam um bom estado nutricional em vitamina $\mathrm{E}$, reflexo de uma ingestão satisfatória dos alimentos fonte como vegetais verde-escuros, oleaginosas e óleos vegetais.

Em síntese, os resultados encontrados apontam uma condição preocupante em relação ao perfil nutricional da lactante e possíveis impactos para a doação de LM. Apesar de entre todos os nutrientes apresentados apenas a vitamina $\mathrm{A}$ ter associação com a concentração no LH, é importante que a nutriz apresente níveis adequados de todos os micronutrientes, para o funcionamento apropriado do seu organismo e para manutenção de bom estado geral da sua saúde, que pode favorecer o processo de doação. Diante disso, destaca-se a importância de realizar ações de educação alimentar e nutricional neste período do ciclo reprodutivo, principalmente no que se refere à qualidade nutricional da dieta, aproveitando o momento em que as mulheres procuram os serviços de saúde para acompanhamento de seus filhos ${ }^{33}$.

\section{Conclusões}

Os achados apontaram que entre as lactantes avaliadas há predominância de adultas jovens, primíparas, nível de escolaridade variado, devido às diferentes regiões estudadas, de baixa renda, com maior propensão ao excesso de peso e carências nutricionais, demandando intervenções direcionadas a fim de favorecer melhoria da saúde da nutriz e oportunizar incremento da doação de LH.

Adicionalmente, a escassez de dados exclusivos das doadoras de LM apontam a necessidade de mais estudos que investiguem as características sociodemográficas e nutricionais desta população, de modo a detectar os fatores associados ao processo de doação e incentivá-lo desde o período pré-natal.

Por fim, salienta-se a importância da atenção integral à saúde da lactante como agente preponderante para a doação de $\mathrm{LH}$ e cuidado com a saúde infantil.

\section{Colaboradores}

Todos os autores contribuíram para a concepção, estruturação, análise e interpretação dos dados, e desenvolveram ou realizaram a revisão crítica do artigo, com o objetivo de obter um conteúdo intelectualcondizente com esta revista. 


\section{Referências}

1. Galvão MTG, Vasconcelos SG, Paiva SDS. Mulheres doadoras de leite humano. Acta Paulista de Enfermagem 2006; 19(2):157-161.

2. Brasil. Agência Nacional de Vigilância Sanitária. Resolução RDC no 171, de 4 de setembro de 2006. Disposição sobre o Regulamento Técnico para o Funcionamento de Bancos de Leite Humano. Diário Oficial da União 2006; 5 set.

3. Wenzel D, Souza SBD. Fatores associados ao aleitamento materno nas diferentes Regiões do Brasil. Revista Brasileira Saúde Materno Infantil 2014; 14(3):241-249.

4. Castro MBTD, Kac G, Sichieri R. Determinantes nutricionais e sociodemográficos da variação de peso no pós-parto: uma revisão da literatura. Revista Brasileira Saúde Materno Infantil 2009; 9(2):125-137.

5. Ferro NDG, Vale IND, Carmona EV, Abrão ACFD. Factors related to unsuccessful lactogenesis - a literature review. Journal Nursing 2009; 8(3):16764285.

6. Azeredo VB, Pereira KB, Silveira CBD, Santos AMCD, Pedruzzi LM. Estado nutricional de nutrizes adolescentes em diferentes semanas pós-parto. Revista Brasileira de Ginecologia Obstétrica 2011; 33(4):176180.

7. Brasil. Ministério da Saúde (MS). PNDS 2006: Pesquisa Nacional de Demografia e Saúde da Criança e da Mulher. Brasília: MS; 2008.

8. Nakamori M, Ninh NX, Isomura H, Yoshiike N, Hien VTT, Nhug BT, Nhien NV, Nakano T, Khan NC, Yamamoto S. Nutritional status of lactating mothers and their breast milk concentration of iron, zinc and copper in rural Vietnam. J Nutr Sci Vitaminol (Tokyo) 2009; 55(4):338-345.

9. Souza G, Saunders C, Dolinsky M, Queiroz J, Campos A, Ramalho A. Vitamin A concentration in mature human milk. J Pediatr (Rio J) 2012; 88(6):496-502.

10. Bachour P, Yafawi R, Jaber F, Choueiri E, Abdel-Razzak Z. Effects of smoking, mother's age, body mass index, and parity number on lipid, protein, and secretory immunoglobulin A concentrations of human milk. Breastfeed Medicine 2012; 7(3):179-188.

11. Cherop C, Keverenge-Ettyang A, Mbagaya GM. Barriers to exclusive breastfeeding among infants aged 0-6 months in Eldoret municipality, Kenya. J Public Health 2009; 6(1):69-72.

12. Dimenstein R, Lira L, Medeiros ACP, Cunha LRF, Stamford TLM. Efeito da suplementação com vitamina E sobre a concentração de alfa-tocoferol no colostro humano. Rev Panam Salud Pública 2011; 29:399-403.

13. Ettyang GA, Lichtenbelt WDVM, Esamai F, Saris WHM, Westerterp KR. Assessment of body composition and breast milk volume in lactating mothers in pastoral communities in Pokot, Kenya, using deuterium oxide. Ann Nutrition Metabolism 2005; 49:110-117.

14. Harit D, Faridi MMA, Aggarwal A, Sharma SB. Lipid profile of term infants on exclusive breastfeeding and mixed feeding: a comparative study. European Journal Clin Nutr 2008; 62(2):203-209.

15. Lubetzky R, Zaidenberg-Israeli G, Mimouni FB, Dollberg S, Shimoni E, Unga Y, Mandel D. Human milk fatty acids profile changes during prolonged lactation: a cross-sectional study. Isr Med Assoc J 2012; 14(1):710.

16. Marın MC, Sanjurjo A, Rodrigo MA, Alaniz MJTD. Long-chain polyunsaturated fatty acids in breast milk in La Plata, Argentina: relationship with maternal nutritional status. Prostaglandins Leukot Essent Fatty Acids 2005; 73(5):355-360.

17. Maru M, Birhanu T, Tessema DA. Calcium, Magnesium, Iron, Zinc and Copper, Compositions of Human Milk from Populations with Cereal and 'Enset'Based Diets. J Health Sci 2013; 23(2):90-97.

18. Olafsdottir AS, Thorsdottir I, Wagner KH, Elmadfa I. Polyunsaturated fatty acids in the diet and breast milk of lactating icelandic women with traditional fish and cod liver oil consumption. Ann Nutrition Metabolism 2005; 50(3):270-276.

19. Qiao Y, Feng J, Yang J, Gu G. The relationship between dietary vitamin A intake and the levels of sialic acid in the breast milk of lactating women. Journal Nutrition Science Vitaminology 2013; 59(4):347-351.

20. Shashiraj, Faridi MMA, Singh O, Rusia U. Mother's iron status, breastmilk iron and lactoferrin-are they related? Eur J Clin Nutr 2006; 60(7):903-908.

21. Szlagatys-Sidorkiewicz A, Martysiak-Zurowska D, Krzykowski G, Zagierski M, Kaminska B. Maternal smoking modulates fatty acid profile of breast milk. Acta Paediatrica. 2013; 102(8):353-359.

22. Tokusoglu O, Tansug N, Aksi t S, Gonuldinc, Kasirga E, Ozcan C. Retinol and -tocopherol concentrations in breast milk of Turkish lactating mothers under different socio-economic status. Int J Food Sci Nutr 2007; 59(2):166-174.

23. Valentine CJ, Morrow G, Pennell M, Morrow AL, Hodge A, Haban-Bartz A Collins K, Rogers LK. Randomized controlled trial of docosahexaenoic acid supplementation in midwestern US Human milk donors. Breastfeed Medicine 2013; 8(1):86-91.

24. Zhixiong SA, Yang JB, Yue Hc, Zhiwei SA, Xianqing ZA, Jinfang FA, Jingguang LD, Yongning WU. Levels of tetrabromobisphenol A, hexabromocyclododecanes and polybrominated diphenyl ethers in human milk from the general population in Beijing, China. Science Total Environ 2013; 452:10-18.

25. Silva RA, Pereira SCL, Silva RCS, Matos DAA, Santos LC. Saúde e nutrição de candidatas à doação de leite humano. Revista Baiana de Enfermagem 2015; 1(1):1222.

26. Dias RDC, Baptista IDC, Gazola S, Rona MSS, Matioli G. Perfil das doadoras do banco de leite humano do Hospital Universitário de Maringá, Estado do Paraná, Brasil. Acta Science Health Science 2006; 28(2):153-158.

27. Santos DT, Vannuchi MTO, Oliveira MMB, Dalmas JC. Perfil das doadoras de leite do banco de leite humano de um hospital universitário. Health Sci 2009; 31(1):1521.

28. Mello-Neto J, Rondo PHC, Oshiiwa M, Morgano MA, Zacari CZ, Domingues S. The influence of maternal factors on the concentration of vitamin A in mature breast milk. Clin Nutr 2009; 28(2):178-181. 
29. Örün ES, Yalçın S, Aykut O, Orhan G, Koç-Morgil G, Yurdakök K, Uzun R. Mercury exposure via breast-milk in infants from a suburban area of Ankara, Turkey. Turk J Pediatr 2012; 54(2):136-143.

30. Colomina GS, Lara NG, Vieco DE, Román SV, Alonso EC, Pallás Alonso CR. Características de las mujeres donantes de un banco de leche materna y relación con el tiempo de donación. An Pediatr (Barc) 2014; 80(4):236-241.

31. Sánchez CL, Rodríguez AB, Sánchez J, González R., Rivero M, Barriga C, Cubero J. Calcium intake nutritional status in breastfeeding women. Arch Latinoamerican Nutrition 2008; 58(4):371-376.

32. Severi C, Hambidge M, Krebs N, Alonso R, Atalah E. Zinc in plasma and breast milk in adolescents and adults in pregnancy and pospartum; a cohort study in Uruguay. Nutricion Hospitalaria 2013; 28(1):223-228.

33. Tavares MP, Devincenzi MU, Sachs A, Abrão ACFV. Estado nutricional e qualidade da dieta de nutrizes em amamentação exclusiva. Acta Paulista de Enfermagem 2013; 26(3):294-298.

34. Quinn EA, Largado F, Power M, Kuzawa CW. Predictors of breast milk macronutrient composition in Filipino mothers. Am Journal Human Biology 2012; 24(4):533540.

35. Antonakou A, Skenderi KP, Chiou A, Anastasiou CA, Bakoula C, Matalas AL. Breast milk fat concentration and fatty acid pattern during the first six months in exclusively breastfeeding Greek women. Eur J Nutr 2013; 52(3):963-973.

36. Damião JDJ. Influência da escolaridade e do trabalho maternos no aleitamento materno exclusivo. Revista Brasileira de Epidemiologia 2008: 11(3):442-452.

37. Brasil. Decreto n. ${ }^{\circ} 6.135$, de 26 de junho de 2007. Dispõe sobre o Cadastro Único para Programas Sociais do Governo Federal e dá outras providências. Diário Oficial da União 2007; 27 jun.

38. Neves LS, Mattar MJG, Sá MVM, Galisa MS. Doação de leite humano: dificuldades e fatores limitantes. Mundo Saúde 2011; 35(2):156-161.

39. Coutinho ACFP, Soares ACO, Fernandes PS. Knowledge of mothers about the benefits of breastfeedinf to women's health. Rev Enferm UFPE 2014; 8(5):12131220.

40. Ribeiro KDDS, Araujo KFD, Souza HHBD, Soares FB, Pereira MDC, Dimenstein R. Nutritional vitamin A status in northeast Brazilian lactating mothers. J Hum Nutr Diet 2010; 23(2):154-161.

41. Lira LQD, Ribeiro PPC, Grilo EC, Freitas JKCO, Dimenstein R. Perfil de retinol no soro e colostro de puérperas atendidas em maternidade pública Brasileira e sua associação com características maternas e obstétricas. Revista Paulista de Pediatria 2011; 29(4):515-520.
42. Lopes AG, Komatsu TR, Asakura L, Sachs A, Silva CVDD, Abrão ACFV, Tavares MP, Coelho LC. Ingestão dietética de cálcio por lactantes em aleitamento materno exclusico. Nutrire 2011; 36(2):33-45.

43. Nogueira JL, Saunders C, Carmo Leal M. Métodos antropométricos utilizados na avaliação da retenção do peso no período pós-parto: uma revisão sistemática. Cien Saude Colet 2015; 20(2):407-420.

44. Institute of Medicine (IOM). Dietary Reference Intakes for Energy, Carbohydrate, Fiber, Fat, Fatty Acids, Cholesterol, Protein, and Amino Acids (Macronutrients). Washington: The National Academies Press; 2005.

45. Valentine CJ, Wagner CL. Nutritional management of the breastfeeding dyad. Pediatric Clin Nutr. 2013: 60(1):261-274.

46. Correia-Santos AM, Bolognini Pereira K, Erthal Santelli R, Teles Boaventura G, Blondet de Azeredo V. Dietary supplements for the lactating adolescent mother: influence on plasma micronutrients. Nutricion Hospitalaria 2011; 26(2):392-398.

47. Soares FB, Ribeiro KDDS, Dimenstein R. Análise do retinol sérico em puérperas atendidas em uma maternidade pública de Natal/RN. RBAC 2008; 40(2):129-131.

48. Ramalho A, Padilha P, Saunders C. Análise crítica de estudos brasileiros sobre deficiência de vitamina A no grupo materno-infantil. Revista Paulista de Pediatria 2008; 26(4):392-399.

49. Brasil. Ministério da Saúde (MS). Manual de condutas gerais do Programa Nacional de Suplementação de Vitamina A. Brasília: MS; 2013.

50. Silva LDSV, Thiapó AP, Souza GGD, Saunders C, Ramalho A. Micronutrientes na gestação e lactação. Revista Brasileira Saúde Materno Infantil 2007; 7(3):237-244.

51. Dimenstein R, Pires JF, Garcia IRS, Lira IGD. Levels of alpha-tocopherol in maternal serum and colostrum of adolescents and adults. Revista Brasileira de Ginecologia e Obstetrícia 2010; 32(6):267-272.

Artigo apresentado em 11/12/2015

Aprovado em 02/03/2016

Versão final apresentada em 04/03/2016 